\title{
Suicidal and self-harm presentations to Emergency Departments: The challenges of identification through diagnostic codes and presenting complaints
}

I-9

(C) The Author(s) 2019

Article reuse guidelines:

sagepub.com/journals-permissions DOI: 10.1 I77//8333583/9857/88 journals.sagepub.com/home/himj

@SAGE

\author{
Jerneja Sveticic, $B S c, M S c P_{s y c h}{ }^{\oplus}$, \\ Nicholas CJ Stapelberg, MBBS, PhD, FRANZCP ${ }^{1,2}$, \\ Kathryn Turner, MBBS, FRANZCP
}

\begin{abstract}
Background: The accuracy of data on suicide-related presentations to Emergency Departments (EDs) has implications for the provision of care and policy development, yet research on its validity is scarce. Objective: To test the reliability of allocation of ICD-IO codes assigned to suicide and self-related presentations to EDs in Queensland, Australia. Method: All presentations due to suicide attempts, non-suicidal self-injury (NSSI) and suicidal ideation between I July 2017 and 31 December 2017 were reviewed. The number of presentations identified through relevant ICD-I0-AM codes and presenting complaints in the Emergency Department Information System were compared to those identified through an application of an evolutionary algorithm and medical record review (gold standard). Results: A total of 2540 relevant presentations were identified through the gold standard methodology. Great heterogeneity of ICD-I0-AM codes and presenting complaints was observed for suicide attempts (40 diagnostic codes and 27 presenting complaints), NSSI (27 and I6, respectively) and suicidal ideation (38 and 34, respectively). Relevant ICD codes applied as primary or secondary diagnosis had very low sensitivity in detecting cases of suicide attempts (18.7\%), NSSI (38.5\%) and suicidal ideation (42.3\%). A combination of ICD-I0-AM code and a relevant presenting complaint increased specificity, however substantially reduced specificity and positive predictive values for all types of presentations. ED data showed bias in detecting higher percentages of suicide attempts by Indigenous persons (10.1\% vs. $6.9 \%$ ) or by cutting ( $28.1 \%$ vs. $10.3 \%)$, and NSSI by female presenters $(76.4 \%$ vs. $67.4 \%)$. Conclusion: Suicidal and self-harm presentations are grossly under-enumerated in ED datasets and should be used with caution until a more standardised approach to their formulation and recording is implemented.
\end{abstract}

\section{Keywords (MeSH)}

clinical coding; data quality; ICD-I0-AM; hospital information systems; health information management; suicide; suicide attempted; self-injurious behavior; suicidal ideation; depression

Supplementary keywords

non-suicidal self-injury; NSSI; self-harm

\section{Background}

Around 800,000 people die by suicide every year worldwide, with estimates of at least 25 times that number engaging in non-fatal suicidal behaviours (WHO, 2014). The most recent available statistics for Australia showed that in 2017, 3128 Australians took their life (Australian Bureau of Statistics, 2018). Emergency Departments (EDs) are important settings for suicide prevention, as they are often the primary point of engagement with health professionals for people at risk of suicide or after a suicide attempt (Da Cruz et al., 2011). Accurate surveillance of suicide attempts and self-harm represents a core element of the public health model of suicide prevention. However, ED administrative datasets vary significantly in completeness and quality, particularly regarding morbidity due to external causes

\footnotetext{
I Gold Coast Health, Australia

2 Bond University, Australia

Accepted for publication May 25, 2019.

Corresponding author:

Jerneja Sveticic, Research Officer, Gold Coast Health, Mental Health and Specialist Services, I Hospital Bvld, Southport, QLD 42I5, Australia.

E-mail: jerneja.sveticic@health.qld.gov.au
} 
(Hedegaard et al., 2018). These inconsistencies hinder the determination of the true rate of suicidal and self-harm presentations to hospital settings at regional or national levels, allocation of appropriate resources and creation of targeted interventions (Barczyk et al., 2018; Spicer and Miller, 2000).

Research on the validity of data on suicidal and selfharm presentations in ED records is scarce (Walkup et al., 2012). Study outcomes from the United Kingdom, United States and Canada suggest that administrative data underestimate the volume of presentations after suicide attempts and self-harm by around $60 \%$, when evaluated against clinical records or assessments of suicidality with standardised tools (Clements et al., 2016; Randall et al., 2017; Stanley et al., 2017). The magnitude of this underenumeration may be particularly high for females and ethnic minorities (Barczyk et al., 2018) and for patients with more complex clinical pictures (Stanley et al., 2017).

Diagnostic codes are often used to inform on performance outcomes (Australian Council on Healthcare Standards, 2017; Boyle et al., 2018). In Australia, different states use different ED coding systems for diagnoses assigned to presentations. Mapping of these codes is done at the national level and collated in the National Non-Admitted Patient Emergency Department Care Database, which produces consistent ED data for research, healthcare and service planning. The suitability of ED discharge diagnoses for monitoring health outcomes has been questioned before (Howell et al., 2014), yet no study to date has evaluated their utility in the context of suicidal and self-harm presentations.

The aim of the present work was to test the reliability of ED coding (specifically, diagnostic codes and presenting complaints) assigned to suicide and self-harm-related presentations to EDs within the Gold Coast Hospital and Health Service (GCHHS) in Queensland, Australia. Analysis of their specificity is pertinent following Queensland Health's recent introduction of a performance indicator monitoring the follow-up of suicidal patients discharged from ED (State of Queensland, 2018). This work also represents an important component of the implementation and evaluation of the GCHHS' Suicide Prevention Strategy (SPS) (Gold Coast Health, 2016). The SPS is based on the Zero Suicide Framework (Covington et al., 2011), a systems approach to suicide prevention within a healthcare setting with an underpinning continuous quality improvement approach. The current challenges surrounding availability of reliable data that can be provided to clinical teams in a timely manner and thus inform further systems improvements represent a significant barrier to this process.

\section{Method}

\section{Context of the study}

This analysis was conducted within the GCHHS, which provides public mental health services for a population of approximately 560,000 people. GCHHS has two EDs that represent the largest primary points of presentation for persons of all ages at risk of suicide. A cohort of suicidal and self-harm-related presentations between 1 July 2017 and 31 December 2017 was examined.

\section{The Emergency Department Information System}

The Emergency Department Information System (EDIS) is an electronic health record system that captures ED attendance data across Queensland. At the point of triage, each presentation is allocated a presenting complaint that best describes the underlying symptoms and further informs diagnosis and management. No international standard exists for presenting complaints entries (Malmström et al., 2012). Across Queensland, a drop-down menu of around 95 presenting complaints is available in EDIS; each is depicted by a unique code and corresponding description.

Upon discharge from the ED, one or more diagnostic codes from the Australian Modification of the International Statistical Classification of Diseases and Health Related Problems, 10th revision (ICD-10-AM) (National Centre for Classification in Health, 2018) are assigned by the discharging clinician. In EDIS systems used across Queensland, a drop-down menu of a limited subset of codes is available, with 28 of those describing psychiatric disorders which include presentations due to suicidal thoughts or behaviours. It is notable that in the ICD-10-AM, suicidal and self-harm behaviours are captured in the range X60-X84; however, in the EDs included in our study, only the code X84 "intentional self-harm by unspecified means" was available as a discharge diagnosis.

The EDIS was used as the primary source of information for identification of suicidal and self-harm presentations within GCHHS. A software program "Searching EDIS for Records of Suicidal Presentations (SERoSP)" was developed in MATLAB to identify relevant cases. It was based on an evolutionary algorithm which weights 136 variables (demographic characteristics, diagnostic codes, presenting complaints, key words appearing in the triage text, treatment received, etc.) from a training dataset of data from 2015, where 10,462 suicidal presentations were identified by a psychiatrist. The program "learned" from trial and error, over 150 successive generations, using 100 variations of variable weights per generation. SERoSP was then run on a psychiatrist-rated validation dataset of 7786 cases from 2017 , using the weights generated by the evolutionary algorithm. Receiver operating characteristics curves were used to determine an optimum scoring cut-off, sensitivity and specificity. Ultimately, SERoSP was shown to have a 95\% sensitivity and $92 \%$ specificity in identifying suicidal and self-harm presentations (Stapelberg et al., 2018) and is currently being used in the GCHSS' evaluation of SPS.

\section{Variables extracted}

For each presentation, the patient's age, sex (male/female), Indigenous background (yes/no), Unit Record Number, date and time of presentation, triage free-text narrative, presenting complaint and primary and secondary diagnoses following ICD-10-AM were extracted. 


\section{Identification of cases using diagnostic codes and presenting complaints}

The ICD-10-AM code X84 "intentional self-harm by unspecified means" was used to identify cases of suicide attempts and self-harm (as this was the only code denoting suicidal and self-harm behaviours in EDIS). The ICD-10AM code R45.81"suicidal ideation" was used to identify cases of suicidal ideation. Relevant cases in all three categories (suicide attempts, self-harm and suicidal ideation) were also identified through the application of the presenting complaint 30005 "suicidal-homicidal ideation."

\section{Gold standard: Medical record review}

A team of trained research officers performed medical record review for the identified presentations using the clinical databases Consumer Integrated Mental Health Application and Electronic Medical Record. The officers reviewed cases independently of each other; however, regular meetings were held between them to ensure a standardised coding process, and a clinical expert assisted in the resolution of more complex cases.

Each presentation extracted by SERoSP was categorised into one of the following groups, using definitions based on previous literature (De Leo et al., 2006) and the World Health Organization's (WHO) Practice Manual for Establishing and Maintaining Surveillance Systems for Suicide Attempts and Self-Harm (WHO, 2016): 1 = not applicable (no evidence of any type of suicidality); 2 = suicidal ideation (including thoughts of suicide, ranging from passive to active, and behaviours such as threats, preparatory acts and interrupted or aborted suicide attempts); $3=$ non-suicidal self-injury (NSSI; self-harming behaviour without intent to die); 4 = suicide attempt (self-harming behaviour with intent to die); or $5=$ ambiguous (inadequate evidence to decide on any of the above categories). Cases categorised as not applicable and ambiguous were excluded from analysis. Frequencies of ICD-10-AM codes and presenting complaints were calculated for all cases identified through the gold standard approach. A range of additional statistics (sensitivity, specificity, positive predictive value [PPV] and negative predictive value [NPV]) were calculated for ICD-10-AM codes X84 and R45.81, and the presenting complaint "suicidal-homicidal ideation," in identifying each type of presentation (suicide attempts, NSSI and suicidal ideation). Demographic information (age, gender, Indigenous status) and methods used in suicide attempts were compared between cases identified through the two approaches using $t$-test and $\chi^{2}$ statistics.

All analyses were performed with SPSS, version 24 .

\section{Ethics}

This work was performed as part of the project Gold Coast Mental Health and Specialist Services Suicide Prevention Strategy: Evaluation. It was recognised as Quality Activity by the GCHSS Human Research Ethics Committee and
Table I. Demographic characteristics of the sample of suicidal and self-harm presentations.

\begin{tabular}{lccc}
\hline & $\begin{array}{c}\text { Suicide } \\
\text { attempt } \\
(N=742)\end{array}$ & $\begin{array}{c}\text { NSSI } \\
(N=187)\end{array}$ & $\begin{array}{c}\text { Suicidal } \\
\text { ideation } \\
(N=16 \mathrm{II})\end{array}$ \\
\hline Age (mean) & $\begin{array}{c}33.3 \\
(\mathrm{SD}=16.0)\end{array}$ & $\begin{array}{c}25.7 \\
(\mathrm{SD}=12.0)\end{array}$ & $\begin{array}{c}33.7 \\
(\mathrm{SD}=15.3)\end{array}$ \\
$\begin{array}{l}\text { Sex } \\
\quad \text { Female }\end{array}$ & $63.9 \%$ & $67.4 \%$ & $46.4 \%$ \\
$\quad \begin{array}{l}\text { Male } \\
\text { Indigenous status } \\
\quad \text { Indigenous }\end{array}$ & $36.1 \%$ & $32.6 \%$ & $53.6 \%$ \\
$\quad$ Not Indigenous & $63.1 \%$ & $3.7 \%$ & $4.3 \%$ \\
\hline
\end{tabular}

NSSI: non-suicidal self-injury.

thus granted a research ethics exemption (LNR/2018/ QGC/47473).

\section{Results \\ Description of sample}

A total of 3417 presentations between July and December 2017 were identified using SERoSP. Of those, 873 were categorised as not applicable, 1611 as suicide ideation, 187 as NSSI, 742 as suicide attempts and 4 cases as ambiguous. Not applicable and ambiguous cases were excluded from further analysis, yielding a total sample of 2540 presentations. Persons presenting with suicide attempts and suicidal ideation had a similar mean age (around 33 years), with presentations due to NSSI being on average younger (mean age 25.7 years). More females than males presented with suicide attempts and NSSI, but the sex ratio was reversed in presentations due to suicidal ideation $(46.4 \%$ were females and $53.6 \%$ were males). The percentage of persons of Indigenous background was the highest among presentations due to suicide attempts $(6.9 \%)$ and lowest among NSSI cases $(3.7 \%)$ (see Table 1$)$.

\section{Distribution of ICD-IO-AM codes and presenting complaints}

Table 2 lists a total of 40 different ICD-10-AM codes used as primary diagnoses for cases of suicide attempts, 27 for cases of NSSI and 38 for cases of suicidal ideation (exact frequencies are shown only for diagnoses assigned to 4 or more cases). The most frequent codes among suicide attempts were T50.9 "other drug poisoning" (20.8\%), followed by $17.5 \%$ of cases with code X84 "intentional self-harm by unspecified means," and $12.0 \%$ with R45.81 "suicidal ideation." Among cases of NSSI, code X84 was allocated to $36.4 \%$ of cases, and code R45.81 to $16.0 \%$ of cases. Among presentations with suicidal ideation, $41.4 \%$ were coded as R45.81, 15.8\% as F43.9 "reaction to severe stress," followed by a substantial percentage of cases for which primary diagnoses denoted various mental illnesses (e.g. depression, psychotic episode, anxiety and personality disorder). 
Table 2. Distribution of ICD-I0-AM codes as primary diagnosis assigned to suicidal and self-harm presentations.

\begin{tabular}{|c|c|c|c|}
\hline ICD-I0-AM code & ICD-I0-AM description & $n$ & $\%$ \\
\hline \multicolumn{4}{|c|}{ Suicide attempts $(N=742)^{\mathrm{a}}$} \\
\hline T50.9 & Other drug poisoning & 154 & 20.8 \\
\hline X84 & Intentional self-harm by unspecified means & 130 & 17.5 \\
\hline R45.8I & Suicidal ideation & 89 & 12.0 \\
\hline T39.1 & Paracetamol poisoning & 88 & 11.9 \\
\hline T42.4 & Benzodiazepine poisoning & 56 & 7.5 \\
\hline T43.9 & Antidepressant poisoning & 50 & 6.7 \\
\hline T43.5 & Antipsychotic poisoning & 24 & 3.2 \\
\hline F32.9 & Depression & 19 & 2.6 \\
\hline F43.9 & Reaction to severe stress & 15 & 2.0 \\
\hline $\mathrm{T} 40.0$ & Opiate toxicity & 14 & 1.9 \\
\hline T44.3 & Anticholinergic poisoning & 10 & 1.3 \\
\hline T7I & Asphyxiation, strangulation, hanging & 10 & 1.3 \\
\hline FI0.0 & Alcohol intoxication & 8 & 1.1 \\
\hline T44.7 & Beta-blocker poisoning & 8 & I.I \\
\hline T43.69 & Amphetamine poisoning & 7 & 0.9 \\
\hline T45.0 & Antiemetic poisoning & 7 & 0.9 \\
\hline FI9.2 & Drug addiction & 5 & 0.7 \\
\hline S5I.9 & Lacerated forearm & 5 & 0.7 \\
\hline T39.3 & Non-steroidal anti-inflammatory poisoning & 5 & 0.7 \\
\hline F29 & Psychotic episode & 4 & 0.5 \\
\hline Z53.2 & Medical advice on medication & 4 & 0.5 \\
\hline \multicolumn{4}{|l|}{$N S S I(N=187)^{b}$} \\
\hline X84 & Intentional self-harm by unspecified means & 68 & 36.4 \\
\hline R45.8I & Suicidal ideation & 30 & 16.0 \\
\hline F43.9 & Reaction to severe stress & 18 & 9.6 \\
\hline S51.9 & Lacerated forearm & 12 & 6.4 \\
\hline F32.9 & Depression & 10 & 5.3 \\
\hline F91.9 & Behavioural problems - child & 7 & 3.7 \\
\hline F60.9 & Personality disorder & 6 & 3.2 \\
\hline T00.9 & Multiple superficial injuries & 4 & 2.1 \\
\hline Z53.2 & Medical advice on medication & 4 & 2.1 \\
\hline \multicolumn{4}{|c|}{ Suicidal ideation $(N=|6| I)^{c}$} \\
\hline R45.8I & Suicidal ideation & 667 & 41.4 \\
\hline F43.9 & Reaction to severe stress & 254 & 15.8 \\
\hline F32.9 & Depression & 172 & 10.7 \\
\hline FI0.0 & Alcohol intoxication & 86 & 5.3 \\
\hline F29 & Psychotic episode & 55 & 3.4 \\
\hline F41.9 & Anxiety & 52 & 3.2 \\
\hline $\mathrm{F} 60.9$ & Personality disorder & 48 & 3.0 \\
\hline F91.9 & Behavioural problems - child & 42 & 2.6 \\
\hline F99 & Mental illness - no diagnosis & 31 & 1.9 \\
\hline $\mathrm{x} 84$ & Intentional self-harm by unspecified means & 29 & 1.8 \\
\hline FI9.9 & Drug-induced mental disorder & 25 & 1.6 \\
\hline F20.9 & Schizophrenia & 20 & 1.2 \\
\hline Z53.2 & Medical advice on medication & 17 & 1.1 \\
\hline FI9.2 & Drug addiction & 15 & 0.9 \\
\hline $\mathrm{Fl} 0.3$ & Alcohol withdrawal syndrome & 13 & 0.8 \\
\hline $\mathrm{F} 3 \mathrm{I} . \mathrm{I}$ & Bipolar affective disorder - manic & 12 & 0.7 \\
\hline Z60.9 & Did not wait & 9 & 0.6 \\
\hline T50.9 & Other drug poisoning & 8 & 0.5 \\
\hline 120.0 & Possible cardiac chest pain & 7 & 0.4 \\
\hline $\mathrm{F} 32.3$ & Depression - psychotic & 5 & 0.3 \\
\hline F4l.0 & Panic attack & 5 & 0.3 \\
\hline $\mathrm{F} 50.0$ & Anorexia nervosa & 5 & 0.3 \\
\hline $\mathrm{F} 3 \mathrm{I} .3$ & Bipolar affective disorder - depressed & 4 & 0.2 \\
\hline T42.4 & Benzodiazepine poisoning & 4 & 0.2 \\
\hline
\end{tabular}

ICD-IO-AM: Australian Modification of the International Statistical Classification of Diseases and Health Related Problems, I0th revision; NSSI: nonsuicidal self-injury.

${ }^{\mathrm{a}}$ ICD-I0-AM codes with frequency of 3 or less: F09, unspecific organic or symptomatic mental disorder; FI0.3, alcohol withdrawal syndrome; F20.9, schizophrenia; F4I.9, anxiety; F50.0, anorexia nervosa; F60.9, personality disorder; F9I.9, behavioural problems - child; SII.8, lacerated neck; S6I.9, lacerated hand or wrist; S83.6, knee sprain/strain; S9I.7, lacerated ankle and foot; TOI.2, multiple lacerations; TI4.6, lacerated tendon and/or muscle; T38.3, other antidiabetic poisoning; T39.0, salicylate poisoning; T45.5, antihistamine poisoning; T46. I, warfarin poisoning; T58, carbon monoxide inhalation; T88.7, other medication side effect.

bICD-10-AM codes with frequency of 3 or less: FI0.0, alcohol intoxication; F19.2, drug addiction; F29, psychotic episode; F32.3, depression - psychotic; F4I.9, anxiety; F99, mental illness - no diagnosis; S3I.I, lacerated abdomen or lower back; S4I.I, lacerated upper arm; S6I.9, lacerated hand or wrist; S7I.I, lacerated thigh; S8I.9, lacerated lower leg; T0I.2, multiple lacerations; TI4.6, lacerated tendon and/or muscle; T42.4, benzodiazepine poisoning; T43.69, amphetamine poisoning; T43.9, antidepressant poisoning; T44.7, beta-blocker poisoning; T50.9, other drug poisoning.

'ICD-I0-AM codes with frequency of 3 or less: B34.9, viral infection; E86, dehydration; F5I.0, insomnia - non-organic; R07.3, non-cardiac chest pain; R55, syncope/collapse; S62.6, fracture of other finger; T00.9, multiple superficial injuries; T39.I, paracetamol poisoning; T40.0, opiate toxicity; T43.69, amphetamine poisoning; T43.9, antidepressant poisoning; T44.3, anticholinergic poisoning; T7I, asphyxiation, strangulation, hanging; Z04.8, examination and observation for other specified reasons. 
Table 3. Distribution of presenting complaints assigned to suicidal and self-harm presentations. ${ }^{\text {a }}$

\begin{tabular}{|c|c|c|c|}
\hline Presenting complaint - code & Presenting complaint - description & $n$ & $\%$ \\
\hline \multicolumn{4}{|l|}{ Suicide attempts $(N=742)^{b}$} \\
\hline 30005 & Suicidal-homicidal ideation & 240 & 32.3 \\
\hline 9038 & Overdose/toxic exposure & 215 & 29.0 \\
\hline 36000 & Symptoms signs & 100 & 13.5 \\
\hline 30000 & Mental health & 89 & 12.0 \\
\hline 21001 & Altered LOC & 17 & 2.3 \\
\hline 9000 & Injury & 14 & 1.9 \\
\hline 9033 & Laceration/skin tear & 14 & 1.9 \\
\hline 31000 & Altered mental state/neurological symptoms & II & 1.5 \\
\hline 30003 & Mood disturbance & 10 & 1.3 \\
\hline 30001 & Addiction/dependency & 6 & 0.8 \\
\hline 9040 & Strangulation/asphyxia & 4 & 0.5 \\
\hline 11002 & Anxiety/agitation & 4 & 0.5 \\
\hline \multicolumn{4}{|l|}{ NSSI $(N=187)^{c}$} \\
\hline 30005 & Suicidal-homicidal ideation & 60 & 32.1 \\
\hline 30000 & Mental health & 49 & 26.2 \\
\hline 9033 & Laceration/skin tear & 22 & 11.8 \\
\hline 9000 & Injury & 12 & 6.4 \\
\hline 11002 & Anxiety/agitation & 10 & 5.3 \\
\hline 36000 & Symptoms signs & 8 & 4.3 \\
\hline 9038 & Overdose/toxic exposure & 7 & 3.7 \\
\hline 30003 & Mood disturbance & 6 & 3.2 \\
\hline \multicolumn{4}{|l|}{ Suicidal ideation $(N=|6|||)^{d}$} \\
\hline 30005 & Suicidal-homicidal ideation & 826 & 51.3 \\
\hline 30000 & Mental health & 379 & 23.5 \\
\hline 30003 & Mood disturbance & 107 & 6.6 \\
\hline 36000 & Symptoms signs & 103 & 6.4 \\
\hline 11002 & Anxiety/agitation & 67 & 4.2 \\
\hline 31000 & Altered mental state/neurological symptoms & 32 & 2.0 \\
\hline 16003 & Hallucinations & 22 & 1.4 \\
\hline 11001 & Aggression & 17 & I. \\
\hline 30001 & Addiction/dependency & 10 & 0.6 \\
\hline 9038 & Overdose/toxic exposure & 5 & 0.3 \\
\hline 17000 & Reviews/admissions & 5 & 0.3 \\
\hline 25006 & Chest pain & 4 & 0.2 \\
\hline
\end{tabular}

NSSI: non-suicidal self-injury; ED: Emergency Department; LOC: level of consciousness; MVC: motor vehicle collision.

${ }^{\text {a }}$ These presenting complaints are in use across Queensland.

bresenting complaints with frequency of 3 or less cases: I, multi-trauma; 90I2, fall; 9036, MVC-driver; II00I, aggression; I6003, hallucinations; I700 I, planned ED recall or review; 17002, admission/transfer; 17004, inpatient team review; 21020 , seizure; 25004, abdo/pelvis/perineal pain; 25006, chest pain; 250II, limb/joint pain; 250I3, neck/throat pain; 360I0, diarrhoea; 360I5, nausea/vomiting.

'Presenting complaints with frequency of 3 or less cases: 9023, penetrating injury; 9029, blunt injury; 9030, burn/scald; I I00I, aggression; I6003, hallucinations; 17005, requesting investigation; 3000I, addiction/dependency; 30004, sleep disturbance.

${ }^{d}$ Presenting complaints with frequency of 3 or less cases: 30004, sleep disturbance; 9000 , injury; 9012 , fall; 9029 , blunt injury; 9033 , laceration/skin tear; 9036, MVC-driver; 17007, social concern (includes child protection); 17008, unplanned ED representation; 2I00I, altered LOC; 21002 , altered sensation; 21020, seizure; 25004, abdo/pelvis/perineal pain; 25005, back pain; 30002, eating disorder; 3200I, altered breathing; 36002, cough/ rhinorrhoea; 36010 , diarrhoea; 36012 , gait disturbance; 360I4, hypertension/hypotension; 360I 5, nausea/vomiting; 360I8, unsettled; 36020, weight gain/ loss.

Table 3 shows that presentations with suicide attempts were allocated a total of 27 different presenting complaints (most frequently 30005 "suicidal-homicidal ideation" in $32.3 \%$ of cases and 9038 "overdose/toxic exposure" in $29.0 \%$. NSSI presentations had 16 different presenting complaints (most frequently, 30005 "suicidalhomicidal ideation" in $32.1 \%$, and 30000 "mental health" in $26.2 \%$ ). Cases with suicidal ideation had 34 different presenting complaints, most commonly 30005 "suicidal-homicidal ideation" (51.3\%) and 30000 "mental health" (23.5\%). As above, only presenting complaints assigned to 4 or more cases are shown with exact frequencies.

\section{Sensitivity, specificity, PPV and NNV}

For calculation of specificity statistics, ICD-10-AM codes used to identify suicidal and self-harm cases were expanded to include secondary diagnoses. The inclusion of X84 as a secondary diagnosis expanded the sample of suicide attempts by 9 cases (from 130 identified through primary diagnosis; increase of $6.9 \%$ ), NSSI by 3 cases (from 68 identified through primary diagnosis; increase of $4.4 \%$ ) and the inclusion of R45.81 as secondary diagnosis increased cases of suicidal ideation by 14 cases (from 667 identified through primary diagnosis; increase of $2.1 \%$ ) (results not tabulated). 
Table 4. Sensitivity statistics for identification of suicidal and self-harm presentations through diagnostic codes and presenting complaints.

\begin{tabular}{|c|c|c|c|c|c|c|}
\hline & \multicolumn{2}{|c|}{ ICD-I0-AM code X84 } & \multicolumn{2}{|c|}{ Presenting complaint SHI } & \multicolumn{2}{|c|}{$\mathrm{X} 84+\mathrm{SHI}$} \\
\hline & Estimate (\%) & $95 \% \mathrm{Cl}(\%)$ & Estimate (\%) & $95 \% \mathrm{Cl}(\%)$ & Estimate (\%) & $95 \% \mathrm{Cl}(\%)$ \\
\hline \multicolumn{7}{|c|}{ Suicide attempt } \\
\hline Sensitivity & 18.7 & $|6.0-2| .7$ & 32.5 & $29.4-36.4$ & 44.6 & $41.0-48.3$ \\
\hline Specificity & 94.0 & $92.8-95.1$ & 50.8 & $48.1-52.8$ & 46.9 & $44.6-49.2$ \\
\hline PPV & 56.3 & $50.4-62.0$ & 21.4 & $27.3-30.9$ & 25.7 & $24.0-27.5$ \\
\hline NPV & 73.7 & 73.0-74.4 & 64.6 & $63.1-66.2$ & 67.3 & $65.5-69.0$ \\
\hline \multicolumn{7}{|l|}{ NSSI } \\
\hline Sensitivity & 38.5 & $31.5-45.9$ & 32.1 & $27.0-41.4$ & 58.8 & $51.4-65.9$ \\
\hline Specificity & 92.6 & $91.4-93.6$ & 54.7 & $52.4-56.5$ & 50.0 & $48.0-52.1$ \\
\hline PPV & 29.1 & $24.6-34.1$ & 5.3 & $4.4-6.5$ & 8.5 & $7.6-9.6$ \\
\hline \multirow[t]{3}{*}{ NPV } & 95.0 & $94.4-95.5$ & 91.0 & $90.0-91.8$ & 93.9 & $92.8-94.8$ \\
\hline & \multicolumn{2}{|c|}{ ICD-I0 code R45.8I } & \multicolumn{2}{|c|}{ Presenting complaint SHI } & \multicolumn{2}{|c|}{$\mathrm{R} 45.8 \mathrm{I}+\mathrm{SHI}$} \\
\hline & Estimate (\%) & $95 \% \mathrm{Cl}(\%)$ & Estimate (\%) & $95 \% \mathrm{Cl}(\%)$ & Estimate (\%) & $95 \% \mathrm{Cl}(\%)$ \\
\hline \multicolumn{7}{|c|}{ Suicidal ideation } \\
\hline Sensitivity & 42.3 & $39.8-44.7$ & 51.4 & $48.9-53.9$ & 69.3 & $67.0-71.6$ \\
\hline Specificity & 86.2 & $83.8-88.3$ & 67.7 & $63.9-70.1$ & 60.8 & $57.6-63.9$ \\
\hline PPV & 84.1 & $81.7-86.2$ & 73.3 & 7I.2-75.3 & 75.3 & $73.7-76.9$ \\
\hline NPV & 46.4 & $45.2-47.6$ & 44.6 & $42.9-45.6$ & 53.4 & $51.2-55.7$ \\
\hline
\end{tabular}

ICD-I0-AM code X84: intentional self-harm by unspecified means; ICD-I0-AM code R45.8I: suicidal ideation; NSSI: non-suicidal self-injury; SHI: suicidalhomicidal ideation; PPV: positive predictive value; NPV: negative predictive value; ICD-I0-AM: Australian Modification of the International Statistical Classification of Diseases and Health Related Problems, IOth revision.

Sensitivity statistics shown in Table 4 were calculated for three different approaches to identifying cases of suicide attempts, NSSI and suicide ideation: through primary or secondary diagnostic codes, through the presenting complaint "suicidal-homicidal ideation" and finally through a combination of diagnostic codes and presenting complaints. When compared to cases identified through gold standard methodology, ICD-10-AM diagnostic codes had low sensitivity in detecting cases of suicide attempts (18.7\%; 95\% CI 16.0-21.7\%), NSSI (38.5\%; 95\% CI $31.5-45.9 \%)$ and suicidal ideation $(42.3 \%$; 95\% CI $39.8-$ $44.7 \%$ ). In comparison, the use of presenting complaint "suicidal-homicidal ideation" had higher sensitivity for suicide attempts $(32.5 \%$; 95\% CI 29.4-36.4\%) and suicide ideation $(51.4 \%$; 95\% CI 48.9-53.9\%) but lower for NSSI presentations $(32.1 \%$; 95\% CI 27.0-41.4\%). In both methods of case identification, PPV values were highest for suicide ideation and lowest for NSSI cases, which reflected the much higher prevalence of cases with suicidal ideation in the total sample. Expanding the criteria of diagnostic codes with the presenting complaint "suicidal-homicidal ideation" increased sensitivity for all types of presentations, bringing the ability to identify true cases of suicide attempts to $44.6 \%$ (95\% CI $41.0-48.3 \%$ ), NSSI to $58.8 \%$ (95\% CI 51.4-65.9\%) and suicide ideation to $69.3 \%(95 \%$ CI $67.0-71.6 \%$ ). However, increased sensitivity significantly reduced levels of specificity, suggesting that a combination of these approaches would inadvertently detect a large number of false negatives.

Table 5 shows a comparison of characteristics of presentations identified through diagnostic codes X84 and
R45.81 (allocated as primary or secondary diagnoses) and through the gold standard methodology. The code X84 was allocated disproportionately to persons of Indigenous background presenting to ED following suicide attempts, when compared to suicide attempts identified through the gold standards $\left(10.1 \%\right.$ vs. $\left.6.9 \% ; \chi^{2}(2)=7.15, p=0.028\right)$. Suicide attempts identified through the X84 code also had an uneven distribution of methods $\left(\chi^{2}(4)=71.79, p \leq 0.001\right)$, capturing more attempts by cutting $(28.1 \%$ vs. $10.3 \%)$ but less presentations due to poisoning $(59.7 \%$ vs. $81.6 \%)$, when compared to the gold standard. Within cases of NSSI, code X84 identified larger number of female presenters than the gold standard $\left(76.4 \%\right.$ vs. $67.4 \%, \chi^{2}(1)=4.32$, $p=0.038)$. No significant differences were observed in relation to presentations with suicidal ideation.

\section{Discussion}

This is the first Australian study into the reliability of ED coding data on suicidal and self-harm presentations, adding to the limited body of international research on this topic. Results revealed marked heterogeneity in allocated primary diagnostic codes and presenting complaints, as well as gross under-enumeration of these events in the ED administrative dataset. ICD-10-AM code X84 correctly identified $18.7 \%$ of suicide attempts and $38.5 \%$ of cases of NSSI presenting to ED, while the code R45.81 identified $42.3 \%$ of cases with suicide ideation. Similar ranges of sensitivity (13.8-65.0\%) and PPV (4.0-100\%) were noted in a systematic review of six administrative datasets from the United States and Canada (Walkup et al., 2012), although 
Table 5. Characteristics of suicidal and self-harm presentations identified through ICD-I0-AM codes and gold standard.

\begin{tabular}{|c|c|c|c|c|c|c|c|c|c|}
\hline & \multicolumn{3}{|c|}{ Suicide attempt } & \multicolumn{3}{|c|}{ NSSI } & \multicolumn{3}{|c|}{ Suicidal ideation } \\
\hline & $\begin{array}{c}X 84 \\
(N=139)\end{array}$ & $\begin{array}{c}\text { Gold } \\
\text { standard } \\
(N=742)\end{array}$ & $P$ & $\begin{array}{c}X 84 \\
(N=7 I)\end{array}$ & $\begin{array}{c}\text { Gold } \\
\text { standard } \\
(N=187)\end{array}$ & $p$ & $\begin{array}{c}\text { R45.8I } \\
(N=68 \mathrm{I})\end{array}$ & $\begin{array}{c}\text { Gold } \\
\text { standard } \\
(N=|6| I)\end{array}$ & $p$ \\
\hline Age (mean) & $\begin{array}{c}31.2 \\
(S D=16.1)\end{array}$ & $\begin{array}{c}33.3 \\
(S D=16.0)\end{array}$ & 0.156 & $\begin{array}{c}23.7 \\
(S D=10.4)\end{array}$ & $\begin{array}{c}25.6 \\
(\mathrm{SD}=11.9)\end{array}$ & 0.235 & $\begin{array}{c}33.1 \\
(S D=15 . I)\end{array}$ & $\begin{array}{c}33.7 \\
(S D=15.4)\end{array}$ & 0.383 \\
\hline Sex & & & 0.847 & & & 0.038 & & & 0.597 \\
\hline Female & $63.3 \%$ & $64.0 \%$ & & $76.4 \%$ & $67.4 \%$ & & $47.1 \%$ & $46.4 \%$ & \\
\hline Male & $36.7 \%$ & $36.0 \%$ & & $23.6 \%$ & $32.6 \%$ & & $52.9 \%$ & $53.6 \%$ & \\
\hline Indigenous status & & & 0.028 & & & 0.422 & & & 0.971 \\
\hline Indigenous & $10.1 \%$ & $6.9 \%$ & & $2.8 \%$ & $4.3 \%$ & & $4.5 \%$ & $4.3 \%$ & \\
\hline Not Indigenous & $89.9 \%$ & $93.1 \%$ & & $97.2 \%$ & $95.7 \%$ & & $95.5 \%$ & $95.7 \%$ & \\
\hline Method & & & $<0.001$ & & & $\mathrm{~N} /$ & IA & & \\
\hline Poisoning & $59.7 \%$ & $81.6 \%$ & & & & & & & \\
\hline Cutting & $28.1 \%$ & $10.3 \%$ & & & & & & & \\
\hline Hanging/strangulation & $4.3 \%$ & $4.5 \%$ & & & & & & & \\
\hline Combination of methods & $6.5 \%$ & $2.8 \%$ & & & & & & & \\
\hline Other & $1.4 \%$ & $0.8 \%$ & & & & & & & \\
\hline
\end{tabular}

ICD-I0-AM code X84: intentional self-harm by unspecified means; ICD-I0-AM code R45.8I: suicidal ideation; Indigenous status: Aboriginal and/or Torres Strait Islander background; ICD-I0-AM: Australian Modification of the International Statistical Classification of Diseases and Health Related Problems, I0th revision.

several methodological variations between studies need to be noted.

Our analysis also tested the sensitivity when expanding diagnostic criteria to include the presenting complaint "suicidal-homicidal ideation." Subsequently, the sensitivity for all three categories increased more than twofold. However, specificity values of around $50 \%$ indicated that half of cases included in the analysis would have been due to non-suicidal complaints. Comparable results were previously reported by Randall et al., (2017), who found that expanding criteria from codes X60-X84 to include events of undetermined intent and accidental poisonings slightly improved the detection of true cases, but at the expense of a reduced specificity and PPV. In future, alternative criteria for identifying suicidal and self-harm presentations could be explored, such as by inclusion of codes denoting poisoning/overdoses (ICD-10-AM T36-T50 “poisoning by drugs, medicaments and biological substances").

The study also identified that the X84 code is currently more likely to be assigned to presentations by persons of Indigenous background, those presenting with suicide attempts by cutting, and NSSI cases by females. A similar skewness of epidemiological data has recently been observed in intentional self-poisoning cases by young females in hospital datasets (McGill et al., 2018). Stanley et al. (2017) concluded that such misrepresentation of particular cohorts is systematic and that certain groups of patients are more likely to have miscoded representations than others (though their observations related to clinical rather than demographic characteristics). This may represent a substantial issue with regards to overrepresentation of Indigenous presentations in ED statistics, for example.

A noteworthy caveat of the present research is the unavailability of codes other than X84 in the identification of relevant cases presenting to $\mathrm{ED}$, which has contributed to the observed incongruities when compared to a gold standard of reviewing medical records. The impact of this on the identification of relevant presentations could be moderated through the allocation of this code as a secondary diagnosis code, yet our study revealed that overall, only around $5 \%$ of suicidal or self-harm presentations to ED had a secondary diagnosis recorded in EDIS. Consequently, it is reasonable to assume that for many presentations, patients' concomitant conditions were more likely to be noted instead of suicidality (Randall et al., 2017).

Other reasons have been proposed for the frequency of miscoded suicidality at ED triage. Primarily, the busy ED environment suffers from the lack of privacy and time constraints, which prevents the necessary mental health assessment to occur at the time of coding (Howell et al., 2014). Furthermore, ED staff do not receive the training required to reliably apply these codes, which is further exacerbated by variations in clinicians' experience (Phillips et al., 2015) and the fact that patients are often reluctant to divulge information due to stigma, fluidity of intent or memory gaps (Berk and Asarnow, 2015; Freedenthal, 2007).

Several limitations need to be noted regarding this research. First, the utilised gold standard methodology may have missed true suicidal or self-harm presentations as per the reported sensitivity and specificity of SERoSP (Stapelberg et al., 2018). An additional caveat was the inability to perform inter-rater reliability analysis, which was partially alleviated through the involvement of the adjudicator with a wealth of clinical expertise and the exclusion of ambiguous cases for which the decision could not be reached. Next, this work did not differentiate between patients hospitalised following their presentation to ED from those discharged directly from ED. The accuracy of data is 
arguably better for admitted patients, as their medical records are coded by clinical coders who are highly trained in the classification of diagnoses. This inevitably creates discrepancies in the accuracy of codes when compared to those available via the coding system utilised in the ED. Furthermore, it is possible that the greater severity of presentations in admitted patients reduces the likelihood for miscoding (Randall et al., 2017). Further analysis is required into the accuracy of ED coding data depending on the assessments undertaken at ED and their outcomes. Last, the generalisability of our findings may be limited as different EDs may have different coding practices that can affect the ability to accurately detect suicidal and self-harm presentations through their administrative datasets (e.g. by having available a larger number of codes to choose from when assigning the most relevant diagnosis).

\section{Conclusion}

Healthcare managers, clinicians and researchers should use ED administrative data on suicidal and self-harm presentations with great caution until a more standardised approach to the formulation and recording of ED diagnosis is implemented at the national level (Howell et al., 2014). Standardisation of clinical coding in the hospital and health service included in this study was scheduled for April 2019. With it, an alternative diagnostic classification set (Systematized Nomenclature of Medicine [SNOMED]) will be introduced in place of ICD. SNOMED codes have been used to extract suicidal and self-harm presentation in other Australian studies (Perera et al., 2018) and while some researchers have argued they improve the quality of information about why people present to EDs (Lawley et al., 2017), their utility for suicidological research remains unknown. There is a need to develop a more sensitive algorithm to improve their identification (Walkup et al., 2012). The SERoSP tool (Stapelberg et al., 2018), used in the development of the gold standard methodology in this study, could be efficiently used to inform healthcare management and resource allocation in the future.

\section{Acknowledgements}

The authors wish to acknowledge Mr Pete Fugelli from Clinical Excellence Division at Department of Health Queensland for his assistance in extracting data from the EDIS database.

\section{Declaration of conflicting interests}

The authors declared no potential conflicts of interest with respect to the research, authorship, and/or publication of this article.

\section{Funding}

The authors received no financial support for the research, authorship, and/or publication of this article.

\section{ORCID iD}

Jerneja Sveticic (D) https://orcid.org/0000-0002-7378-3582

\section{References}

Australian Bureau of Statistics (2018) 3303.0 - Causes of Death, Australia, 2016. 26 September 2018 ed. Canberra: Australian Bureau of Statistics.

Australian Council on Healthcare Standards (ACHS) (2017) Australasian Clinical Indicator Report: 2009-2016: 18th Edition. Sydney, NSW: ACHS.

Barczyk AN, Piper K, Duzinski SV, et al. (2018) Youth suicide attempt nomenclature used in two central Texas hospitals. Crisis 39(6): 461-468.

Berk MS and Asarnow JR (2015) Assessment of suicidal youth in the emergency department. Suicide and Life-Threatening Behavior 45(3): 345-359.

Boyle S, Dennehy R, Healy O, et al. (2018) Development of performance indicators for systems of urgent and emergency care in the Republic of Ireland: systematic review and consensus development exercise. HRB Open Research 1: 6-17.

Clements C, Turnbull P, Hawton K, et al. (2016) Rates of selfharm presenting to general hospitals: a comparison of data from the multicentre study of self-harm in England and hospital episode statistics. BMJ Open 6(2): e009749.

Covington D, Hogan M, Abreu J, et al. (2011) Suicide Care in Systems Framework. National Action Alliance: Clinical Care \& Intervention Task Force, Washington, D.C., US.

Da Cruz D, Pearson A, Saini P, et al. (2011) Emergency department contact prior to suicide in mental health patients. Emergency Medicine Journal 28(6): 467-471.

De Leo D, Burgis S, Bertolote JM, et al. (2006) Definitions of suicidal behavior: lessons learned from the WHO/EURO Multicentre Study. Crisis 27(1): 4-15.

Freedenthal S (2007) Challenges in assessing intent to die: Can suicide attempters be trusted? OMEGA-Journal of Death and Dying 55(1): 57-70.

Gold Coast Health (2016) Gold Coast Mental Health Suicide Prevention Strategy 2016-2018. Southport: Gold Coast Hospital and Health Services.

Hedegaard H, Schoenbaum M, Claassen C, et al. (2018) Issues in developing a surveillance case definition for nonfatal suicide attempt and intentional self-harm using International Classification of Diseases, Tenth Revision, Clinical Modification (ICD-10-CM) Coded Data. National Health Statistics Reports 108: 1-19.

Howell SC, Wills RA and Johnston TC (2014) Should diagnosis codes from emergency department data be used for case selection for emergency department key performance indicators? Australian Health Review 38(1): 38-43.

Lawley M, Truran D, Hansen D, et al. (2017) SnoMAP: pioneering the path for clinical coding to improve patient care. Studies in Health Technology and Informatics 239: 55-62.

Malmström T, Huuskonen O, Torkki P, et al. (2012) Structured classification for ED presenting complaints - from free text field-based approach to ICPC-application. Scandinavian Journal of Trauma, Resuscitation and Emergency Medicine 20(1): 76.

McGill K, Hiles SA, Handley TE, et al. (2018) Is the reported increase in young female hospital-treated intentional self-harm real or artefactual? Australian \& New Zealand Journal of Psychiatry. DOI: $10.1177 / 0004867418815977$. 
National Centre for Classification in Health (NCCH) (2018) The International Statistical Classification of Diseases and Related Health Problems. Australian modification, version 10 (ICD-10-AM). Sydney, NSW: NCCH.

Perera J, Wand T, Bein KJ, et al. (2018) Presentations to NSW emergency departments with self-harm, suicidal ideation, or intentional poisoning, 2010-2014. Medical Journal of Australia 208(8): 348-353.

Phillips G, Gerdtz MF, Elsom SJ, et al. (2015) Mental health nurses' dispositional decision-making for people presenting to the emergency department with deliberate self-harm: an exploratory study. Perspectives in Psychiatric Care 51(2): 148-153.

Randall JR, Roos LL, Lix LM, et al. (2017) Emergency department and inpatient coding for self-harm and suicide attempts: validation using clinician assessment data. International Journal of Methods in Psychiatric Research 26(3): e1559.

Spicer RS and Miller TR (2000) Suicide acts in 8 states: incidence and case fatality rates by demographics and method. American Journal of Public Health 90(12): 1885-1891.

Stanley B, Currier GW, Chesin M, et al. (2017) Suicidal behavior and non-suicidal self-injury in emergency departments underestimated by administrative claims data. Crisis 39(5): 318-325.

Stapelberg NCJ, Sveticic J and Turner K (2018) Searching EDIS for records of suicidal presentations using an evolutionary algorithm (Abstract). In: Gold Coast Health Research Week Conference (ed Brandenburg C), Gold Coast, Australia, 14-15 November 2018, p. 27. Gold Coast: Gold Coast Health.

State of Queensland (2018) Monitoring and Intervention Plan for Measurement Strategy for 'Connecting Care to Recovery 2016-2021: A Plan for Queensland's State-funded Mental Health, Alcohol and Other Drug Services. Brisbane: Queensland Health.

Walkup JT, Townsend L, Crystal S, et al. (2012) A systematic review of validated methods for identifying suicide or suicidal ideation using administrative or claims data. Pharmacoepidemiology and Drug Safety 21: 174-182.

World Health Organization (WHO) (2014) Preventing Suicide: A Global Imperative. Geneva: WHO.

World Health Organization (WHO) (2016) Practice Manual for Establishing and Maintaining Surveillance Systems for Suicide Attempts and Self-harm. Geneva: WHO. 